\title{
Diseño y construcción de un sistema para medir desgaste en forros de frenos de disco
}

\section{Design and construction of a wear-measuring system in disc brake linings}

Jorge Luis Zambrano Guadalupe. ${ }^{1}$, Mariana Elizabeth Villa Orozco. ${ }^{2}$, Luis Fernando Buenaño Moyano ${ }^{3} \&$ Juan Carlos Palacios Almache. ${ }^{4}$

\begin{abstract}
.
DOI: https://doi.org/10.33262/cienciadigital.v9i2.424

The project was headed to the study, design and construction of an electronic control system to measure the wear on the linings of brake pads in a racing car. An analysis of the problem that originates in brake discs when suffering premature and irreparable damages due to the wear of warning system that brake pads have incorporated, being these the causes of the problem. The selection and implementation of electronic components was done to solve the problem with the brake discs, ensuring their performance, their respective care, increasing the usable life of both the disc and the lining of the brake pads and especially to ensure the physical integrity of the vehicle. The results of the tests were excellent, as they managed to consume $15 \%$ more than the amount of lining that's normally achieved with a steel sheet (strip) and avoids damages in the brake discs surface. By the calculations of wear it is determined that the liners do not affect in any way the incorporated, since similarly measures the wear the same system suffers, ensuring the normal operation and the certainty that you can use any type of brake linings.
\end{abstract}

Key words: Liners, disc, break, wear, electronic.

${ }^{1}$ Escuela Superior Politécnica de Chimborazo, Riobamba, Ecuador,jorge_z1986@hotmail.com

2 ITS Carlos Cisneros, Riobamba, Ecuador, elizabethmariany@hotmail.com

3 Escuela Superior Politécnica de Chimborazo, Riobamba, Ecuador, lfbuenanio@espoch.edu.ec

4 Petroamazonas, Riobamba, Ecuador, juank2981@hotmail.com 


\section{Resumen.}

Este proyecto se encaminó al estudio, diseño y construcción de un sistema de control electrónico para medir el desgaste en los forros de las pastillas de frenos en un automóvil de competición. Se realizó un análisis sobre el problema que se origina en los discos de freno al sufrir daños prematuros e irreparables debido a los sistemas de alerta que traen incorporados, siendo los causantes del problema. Se realizó la selección e implementación de componentes electrónicos para solucionar el problema con los discos de frenos, garantizando su desempeño, su cuidado respectivo, el incremento de la vida útil tanto del disco como del forro de las pastillas de freno y sobre todo velar por la integridad del piloto del vehículo. Los resultados obtenidos en las pruebas fueron excelentes, se logró consumir $15 \%$ más cantidad de forros de lo que normalmente se logra con la lámina de acero y evitar daños en la superficie de los discos. Mediante los cálculos de desgaste se determina que los forros no afectan al sistema incorporado ya que de igual manera mide el desgaste que sufren los mismos, garantizando su normal funcionamiento y la certeza de poder utilizar cualquier tipo de forros de frenos.

Palabras clave: Forros, disco, freno, desgaste, electrónico.

\section{Introducción.}

El sistema de frenos está diseñado para detener el vehículo a voluntad del conductor, mediante el funcionamiento de los diferentes elementos que lo conforman, ayudado por un fluido para amplificar la presión ejercida por el conductor, logrando detener al vehículo con el mínimo esfuerzo posible.

Cabe mencionar que dentro de los sistemas convencionales de frenos de discos que tiene un vehículo de competición, existen dos elementos primordiales los cuales se encargan de realizar la detención del vehículo mediante la fricción existente entre ellos, siendo estos los forros de las pastillas y los discos de frenos.

Se tiene presente que los elementos a sufrir desgaste son los forros de las pastillas de frenos, quienes poseen un límite de desgaste para evitar daños en la superficie de contacto de los discos de frenos.

Este límite lo controla una lámina de acero (fleje), que traen adheridas las pastillas de frenos a sus cuerpos, emitiendo una señal ruidosa al momento de ponerse en contacto con la superficie del disco de freno. 
Algunos automóviles de competición aún poseen dichos sistemas de alerta de desgaste, pero gracias a la tecnología que día a día gana importancia en el mundo del automovilismo se han instalado nuevos dispositivos electrónicos para mejorar la seguridad del sistema de frenos de discos. Dando origen a los sensores de desgaste, para que suplanten a las tradicionales láminas de acero que traen incorporadas las pastillas de frenos, para medir el desgaste que sufren las mismas.

En la actualidad los vehículos de competición poseen varios sistemas que alertan del desgaste en los forros de las pastillas de frenos siendo estos: láminas de acero, los cables conductores y los sensores de desgaste, tratando de mejorar día a día el cuidado de los discos de freno.

Después de haber analizado todos estos sistemas ya incorporados en distintos vehículos de competición y haber estudiado las condiciones de funcionamiento de cada uno de ellos, así como también las exigencias que los circuitos de competición poseen, se implementara un sistema de control electrónico para medir el desgaste de los forros de las pastillas de frenos.

\section{Desarrollo}

Para desarrollar el sistema de control de desgaste se aplicó el método sistémico, el cual impone seguir una secuencia de pasos los cuales conllevaron a modelar el sistema mediante la determinación de sus componentes, así como las relaciones entre ellos formando su estructura y dinámica basadas en un orden establecido por reglas, llegando a la absoluta comprensión de la situación.

La secuencia de pasos que se estableció para desarrollar el sistema de control de desgaste de los forros de las pastillas de freno es:

1. Medir el desgaste de los forros, por medio de un sensor.

2. Convertir la señal enviada por el sensor, por medio de una tarjeta electrónica.

3. Procesar la información, por medio del módulo de control programado.

4. Entregar la información del desgaste, por medio de los actuadores.

5. Verificar y comprobar el desgaste producido en los forros. 
Gráfico 1. Método sistémico aplicado para el desarrollo del sistema

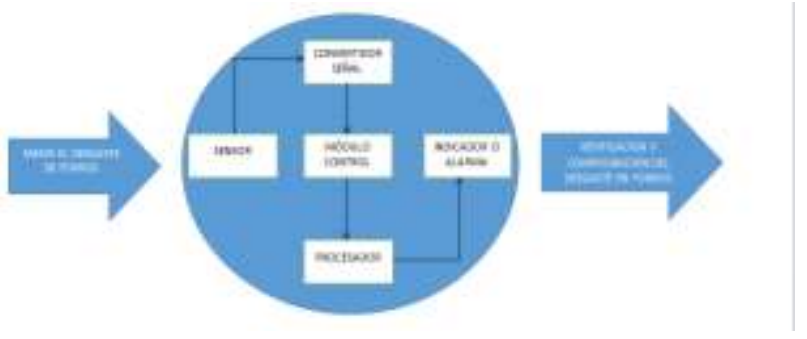

\section{Esquema operativo de control de desgaste.}

En el siguiente diagrama de bloques se indica la secuencia operacional de trabajo para controlar el desgaste que se genera en los forros de las pastillas de frenos con cada frenada que realice el vehículo.

Gráfico 2. Esquema operativo de monitoreo

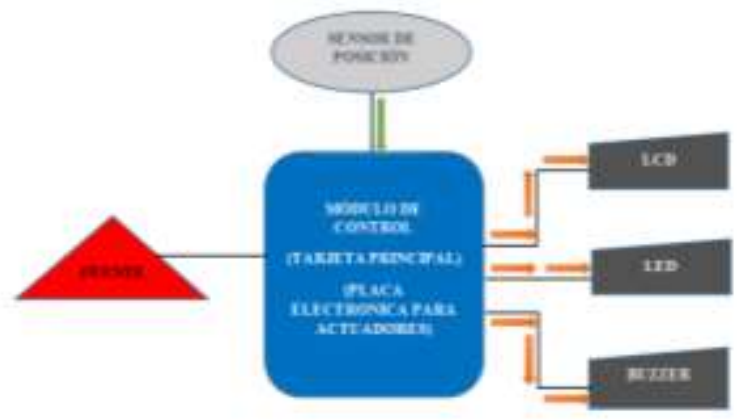

\section{Diseño del módulo de control.}

Para diseñar el módulo de control, se estableció una secuencia de operación, definiendo los parámetros de diseño y construcción, así como también la selección de los distintos elementos que intervendrán en la elaboración del módulo de control electrónico.

Gráfico 3. Diagrama de bloques de la secuencia operacional

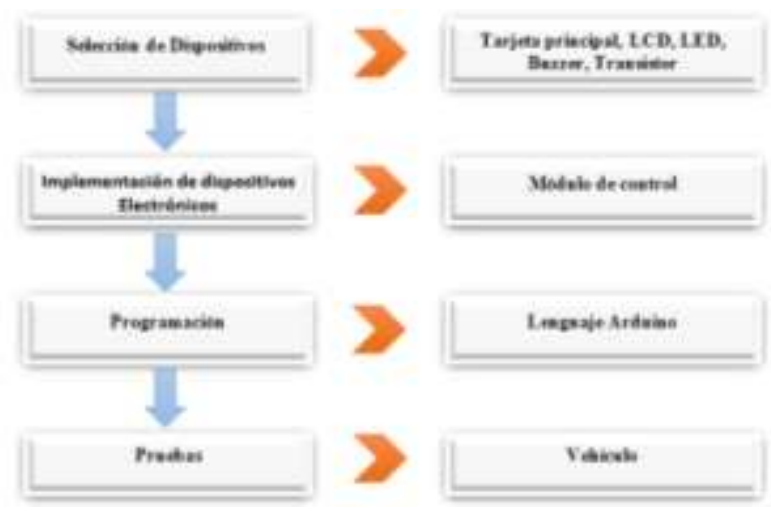




\section{Programación del módulo de control electrónico.}

La programación del módulo de control electrónico se la realizó de acuerdo a las características técnicas y básicas de funcionamiento del sensor de posición de la válvula EGR, utilizando leguaje de Arduino, mediante el software de programación denominado Arduino/Genuino Uno en COM1.

El sensor de posición de la válvula EGR, es la parte física más importante del sistema de medición, esto debido a que las condiciones y características de funcionamiento que este posee son las más apropiadas, teniendo en cuenta las exigencias que el sistema de frenos de discos de un vehículo de competición requiere, siendo estas: resistencia al funcionamiento a la temperatura de trabajo, vibraciones, reacciones químicas y los factores climáticos del medio, para los cuales este sensor es capaz de soportar.

Para la programación fue indispensable la determinación de los pines de conexión y el análisis de los datos que provienen del sensor los cuales permitieron plantear la ecuación matemática, en función del voltaje (x) con respecto al desplazamiento (y) del vástago del sensor, ya que el voltaje resultante depende directamente de la variación del desplazamiento que realiza el vástago.

Gráfico 4. Curva característica obtenida del sensor EGR

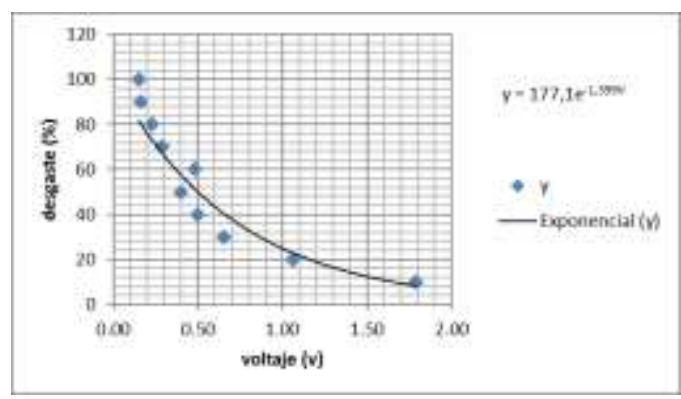

La ecuación resultante es la siguiente:

$$
Y=177.1 e^{-1.399 x}
$$

Esta expresión, evalúa el comportamiento del voltaje provisto por el sensor, resultado de la medición de deformación de la pastilla de freno en el vehículo producto de la medición del desplazamiento del vástago del sensor, solidario al desgaste del material de freno. 
La lógica de la programación está dada de tal forma de que un cumpla diagrama de flujo que rija todo el proceso adecuado de medición ya en el vehículo de competición, el cuál se puede observar en el gráfico 5.

Gráfico 5. Diagrama de flujo de la programación

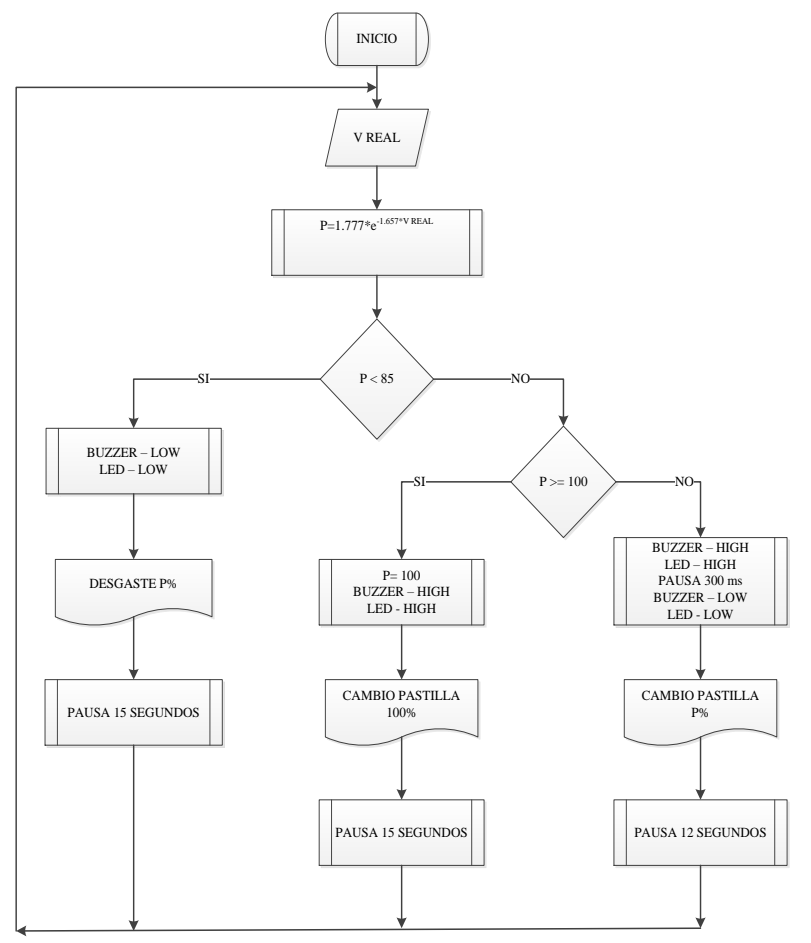

Fuente: Autor

Una vez terminada la programación se procedió a la construcción del módulo de control electrónico y al desarrollo de las partes y piezas que constituyen la parte mecánica del sistema de control, entre ellas se encuentran:

Placa soporte. Esta placa sirve tanto de apoyo como de sujeción del sensor de posición a la mordaza de freno.

Perno de sujeción. Se construyó un perno el cual servirá de sujeción para la mordaza y también como base de apoyo para el sensor de posición desde la cual podrá tomar las diferentes lecturas sobre el desgaste que sufren las pastillas de freno. 
Modificación de mordaza. La modificación a la mordaza de freno se realizó de acuerdo al requerimiento del funcionamiento tanto del sensor de posición como del accionamiento de la mordaza.

Para la construcción de la unidad de control se seleccionaron los componentes adecuados, procediendo luego a la instalación del cableado y conexiones en el vehículo, así como de los componentes mecánicos del sistema.

En cuanto a la afectación de la masa que se incorpora al mecanismo la masa original del sistema del mecanismo de frenos delanteros sin el sistema es de 9,32 $\mathrm{Kg}$ mientras que con el sistema de control adaptado es de $9,983 \mathrm{Kg}$, dando una diferencia de $0.663 \mathrm{Kg}$ lo cual no afecta significativamente al desempeño del sistema.

\section{Resultados}

Dado que el módulo de control cuenta con una pantalla LCD y un buzzer de alerta, este es capaz de monitorear el desgaste y alertar de valores críticos de desgaste de pastillas.

Gráfico 6. Verificación de funcionamiento del sistema

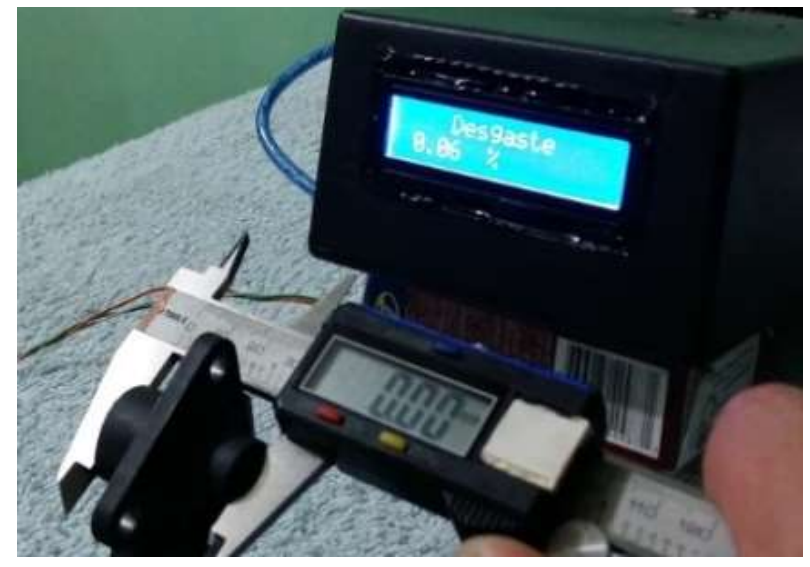

Fuente: Autores

Los resultados de medidas arrojados por el sistema, durante pruebas experimentales se listan en la tabla 1. 
Tabla 1. Medidas de desgaste en mm y valor porcentual que el módulo arroja.

\begin{tabular}{cc}
\hline Porcentaje de desgaste (\%) & Desgaste (mm) \\
\hline $0 \%$ & $0 \mathrm{~mm}$ \\
$10 \%$ & $2 \mathrm{~mm}$ \\
$20 \%$ & $3 \mathrm{~mm}$ \\
$30 \%$ & $4 \mathrm{~mm}$ \\
$40 \%$ & $5 \mathrm{~mm}$ \\
$50 \%$ & $6 \mathrm{~mm}$ \\
$60 \%$ & $7 \mathrm{~mm}$ \\
$70 \%$ & $8 \mathrm{~mm}$ \\
$80 \%$ & $9 \mathrm{~mm}$ \\
$90 \%$ & $10 \mathrm{~mm}$ \\
$100 \%$ & \\
\hline
\end{tabular}

Una vez instalado el sistema, se procedió a realizar pruebas del funcionamiento utilizando pastillas de freno en diversas condiciones, dando el siguiente resultado.

Tabla 2. Resultados de las pruebas

\begin{tabular}{|l|c|l|}
\hline \multicolumn{2}{|c|}{ Pastillas Nuevas } \\
\hline \multicolumn{1}{|c|}{ Denominación } & Resultados & Descripción \\
\hline Medición sensor de desgaste & $0 \mathrm{~mm}$ & Totalmente cerrado. \\
\hline$\%$ de desgaste. & $0 \%$ & Pastillas nuevas \\
\hline Mensaje informativo LCD & Desgaste & $\begin{array}{l}\text { Cantidad desgaste } \\
\text { existente de }\end{array}$ \\
\hline
\end{tabular}


Vol. 3, N², p. 435-446, abril - junio, 2019

\begin{tabular}{|l|c|l|}
\hline \multicolumn{3}{|c|}{ Pastillas usadas y en condiciones de ser sustituidas } \\
\hline $\begin{array}{l}\text { Sensor de Posición (apertura del } \\
\text { vástago). }\end{array}$ & $9 \mathrm{~mm}$ & Alerta el desgaste. \\
\hline$\%$ de desgaste. & $85 \%-100 \%$ & Pastillas al límite del cambio. \\
\hline Mensaje informativo LCD & $\begin{array}{l}\text { Cambio } \\
\text { pastilla }\end{array}$ & $\begin{array}{l}\text { Cambio inmediato de } \\
\text { pastillas. }\end{array}$ \\
\hline
\end{tabular}

\section{Discusión}

La implementación de este instrumento de medición de pastillas de freno para vehículos de competición, en este caso para el caso del Volkswagen Polo en el cual fue instalado, y de acuerdo a las comparaciones con otro tipo de avisadores de desgaste, ha demostrado ser efectivo e indispensable en el mundo competitivo.

Mientras que dispositivos avisadores de desgaste causan daños a los discos de freno de competición ya que al ser normalmente los de fleje los más usados, estos arruinan los discos del vehículo además de que ellos solo dan una idea del desgaste de estos ya, tomando en cuenta además que muchas veces el mecanismo desaprovecha en parte todavía el material de fricción de la pastilla.

En contraparte el dispositivo implementado presenta gráficamente y en porcentaje el desgaste que las pastillas van teniendo a lo largo de su período de vida útil, previniendo daños al disco de freno ya que estos no son invasivos respecto al funcionamiento del sistema de frenos.

Los datos experimentales de acuerdo a las pruebas realizadas en nueve carreras, demostró porcentajes de error de medición de no más del 10\%, el cual no es muy apreciable ya que hay que considerar variables tales como temperatura de funcionamiento y el contexto operacional en el cual se está desarrollando la válida.

Además, en los valores críticos de desgaste la exactitud del sistema es alta e ideal ya que es el momento más crítico en el cual el equipo de carreras desea saber para cambiar las pastillas.

Es importante destacar que luego de las nueve carreras se logró usar un juego de discos de frenos, normalmente se suelen utilizar hasta un juego de discos en tres carreras, además de 
haber utilizado eficazmente el material de fricción hasta que el sistema indicaba la alerta de cambio.

La utilización de este sistema en el equipo de rally en el que se implemento fue exitoso ya que se consiguió un $66.67 \%$ en ahorro en costos de mantenimiento gracias al no tener que usar demasiados pares de discos de freno.

Tabla 3. Análisis Costo beneficio

\begin{tabular}{|c|c|c|c|c|}
\hline $\begin{array}{c}\text { Número } \\
\text { de } \\
\text { Carreras }\end{array}$ & $\begin{array}{c}\text { Número de Juegos de } \\
\text { discos de freno }\end{array}$ & $\begin{array}{c}\text { Sistema de alerta de } \\
\text { pastillas }\end{array}$ & Costo & \% Gasto \\
\hline 9 & 3 & Convencional & 2400 & $66,67 \%$ \\
\hline 9 & 1 & $\begin{array}{c}\text { Sistema de Control } \\
\text { Electrónico }\end{array}$ & 1200 & $33,33 \%$ \\
\hline
\end{tabular}

\section{Conclusiones.}

- Mediante el diseño y construcción del sistema de control electrónico, se controló el desgaste que sufren los forros de las pastillas de frenos, para realizar la sustitución correspondiente, cuando ya han alcanzado su límite máximo de desgaste, establecido por la programación del módulo de control electrónico.

- Se solucionó el problema que existía con los antiguos elementos que alertaban sobre el desgaste de los forros de las pastillas, los cuales provocaban daños en la superficie de los discos de frenos.

- Mediante el análisis de costo beneficio realizado, se comprobó que, al instalar el sistema de control electrónico de desgaste, se obtiene un ahorro del 66,67\% en el costo de mantenimiento del sistema de frenos.

\section{Referencias. Bibliográficas}

Creus, M. (2011). Seguridad e higiene en el trabajo, un enfoque integral. Buenos Aires: Alfaomega. 
Mantaras Daniel, P. L. (2008). Ingeniería del automóvil. Sistemas y comportamiento dinámico. Madrid, España: Thomson.

Galabarro, H. R. (s.f.). ingemecánica. Recuperado el 14 de enero de 2016, de http://ingemecanica.com/tutorialsemanal/tutorialn74.html\#introduccion

Giraldo, A. (2008). Seguridad industrial. México: Copycenter.

Instituto ecuatoriano de seguridad social. (2010). Guía básica de información de seguridad y salud en el trabajo. Quito.

Instituto ecuatoriano de seguridad social. (2010). Reglamento de seguridad y salud de los trabajadores y mejoramiento del medio ambiente de trabajo. Quito.

Luque P, A. D. (2008). Ingeniería del automóvil: sistemas y comportamiento dinámico. Madrid: Paraninfo.

Martínez, D. H. (2000). Manual práctico del automóvil, reparación, mantenimiento y prácticas. Madrid, España: Cultural, S. A.

Martínez, D. H. (2001). Los frenos. En "Manual del automóvil reparación y mantenimiento" (págs. 108-143). Madrid, España: Cultural, S. A.

Ramírez, R. (1992). Manual de seguridad industrial. México: Limusa.

Riofrío Pérez, L. A. (2009). "Implementacion de un sistema de freno de mano, con accionamiento hidráulico para un vehículo suzuki forsa de competición". Latacunga: T-ESPEL-0574.pdf.

ROAD, o. t. (s.f.). On the road. Recuperado el 7 de mayo de 2016, de http://ontheroadmexico.blogspot.com/p/frenos-de-disco.html

Robbins, H. (1993). Manual de seguridad y primeros auxilios. México: Alfaomega.

Toledo, L. C. (9 de enero de 2010). Preparación de vehículos de alto rendimiento. 


\section{PARA CITAR EL ARTÍCULO INDEXADO.}

Zambrano J., Villa M., Buenaño L.\& Palacios J., (2019). Diseño y construcción de un sistema para medir desgaste en forros de frenos de disco. Revista electrónica Ciencia Digital 3(2), 435-446. Recuperado desde:

http://cienciadigital.org/revistacienciadigital2/index.php/CienciaDigital/article/view/424/96 $\underline{8}$

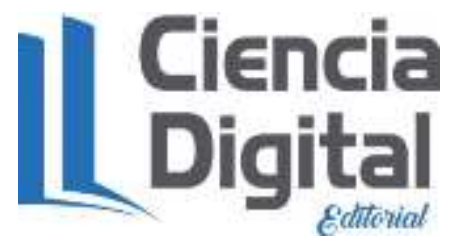

El artículo que se publica es de exclusiva responsabilidad de los autores y no necesariamente reflejan el pensamiento de la Revista Ciencia Digital.

El artículo queda en propiedad de la revista y, por tanto, su publicación parcial y/o total en otro medio tiene que ser autorizado por el director de la Revista Ciencia Digital.
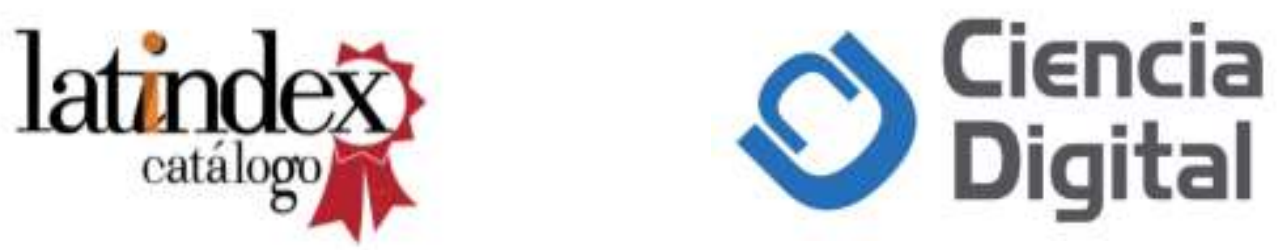